\title{
Entrevista al Sr. Vicepresidente del Área Institucional de la Universidad Nacional de La Plata, Dr. Fernando Tauber
}

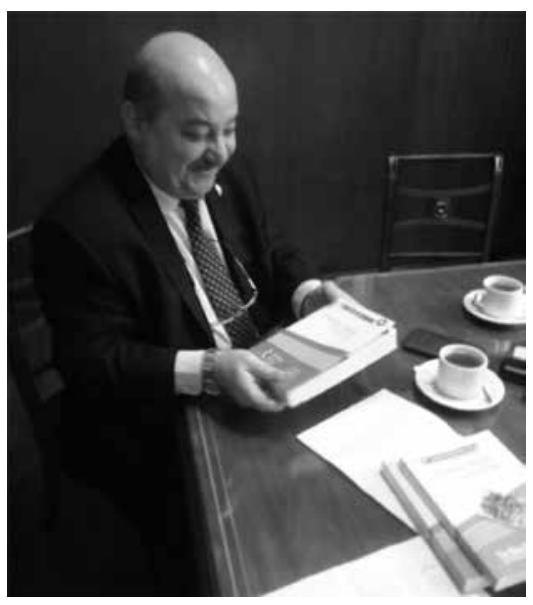

"El capital humano formado es el capital más importante que puede producir la universidad como una institución que produce conocimiento, porque producir conocimiento es producir soberanía".

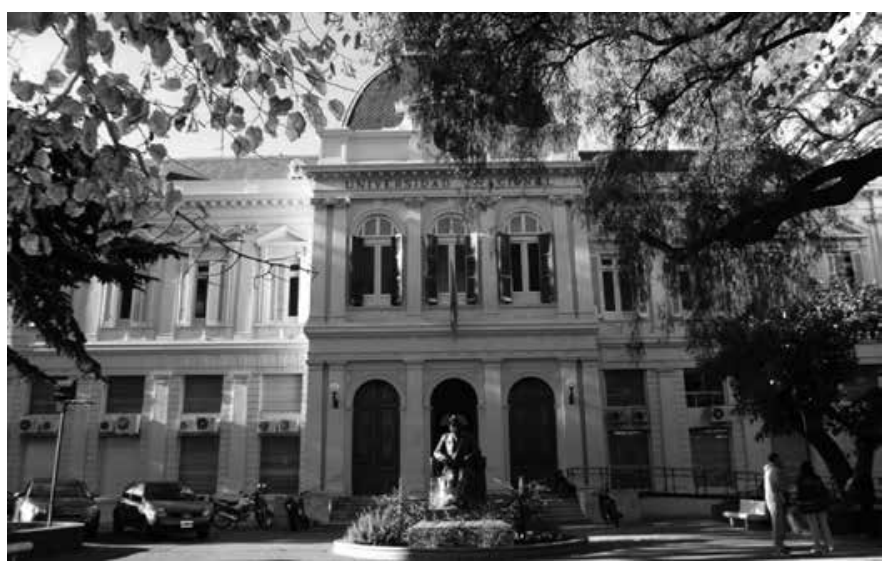


Editores de Revista Derechos en Acción (ReDeA) se reunieron en la sede del rectorado de la Universidad Nacional con el Dr. Fernando Tauber, quien se mostró interesado por la labor que viene desarrollando nuestra publicación. En el marco de ese encuentro le formulamos al Vicepresidente de la UNLP algunas preguntas que transcribimos a continuación.

Redea: El próximo año se cumple un siglo de la histórica reforma universitaria de 1918, aquel movimiento estudiantil de Córdoba que se levantó contra las prácticas autoritarias y dogmáticas de quienes dirigían la Universidad. ¿Cuál es su reflexión actual, cien años después, acerca de la vigencia de aquellos postulados?

La reforma del 18 fue la última gran revolución universitaria que existió en el mundo. La primera había sido el nacimiento propio de la universidad en el Siglo XI, con la universidad de Bolonia, esa primera universidad creada por estudiantes con su primer rector estudiante. La institución universitaria, de por sí, fue un hecho revolucionario, encuentra en una institución una síntesis de donde poner el conocimiento que la humanidad venía produciendo hasta ese momento.

La segunda gran revolución fue la universidad de Berlín, allá en 1810, con Humboldt creándola, e incorporándole a la enseñanza el sentido de la investigación, es decir, la producción de conocimiento que potenciaba la transmisión de conocimiento y viceversa: eso, entre medio de las dos revoluciones industriales, produjo la potencia alemana que nos acompañó hasta muy avanzado el siglo pasado.

Y la tercera revolución fue esta que nace en Córdoba y se extiende por América latina, que rápidamente toma nuestra universidad, en forma casi simultánea, y que incorpora a la enseñanza y a la investigación la extensión universitaria que redondea el modelo universitario de hoy: enseñanza, investigación y extensión. 
Es bueno entenderlo, donde fue apareciendo cada uno de los pilares de la universidad: le da un sentido humanista, consolida la autonomía, el cogobierno, la libertad de cátedra, el acceso a las cátedras por concurso, a la extensión universitaria, la gratuidad de la enseñanza, el acceso irrestricto, transformar la universidad en el espacio natural de debate de los temas que le preocupan a la sociedad. Esos fueron los postulados de la reforma del 18. Como verán, todos tienen una particular vigencia, después paso todo un siglo largo, para que algunos de ellos se fueran consolidando. Hoy la autonomía universitaria es un tema naturalizado en las universidades públicas de la argentina, no tan naturalizado en el resto de las universidades de América latina. Es un tema que defendemos diariamente como defendemos la democracia y custodiamos como custodiamos la democracia.

La gratuidad de la enseñanza y el ingreso irrestricto lo conseguimos con una Ley del año 2015, al final del gobierno anterior. Es decir, la gratuidad pendía de un decreto presidencial de Perón. Perón fue un presidente que tuvo una mala relación con las universidades públicas, pero tenía una visión sobre el desarrollo nacional y entendía que la educación superior era una herramienta fundamental del desarrollo. Por eso creó las universidades populares, luego las tecnológicas, las mayores dedicaciones a la investigación y le dió definitivamente gratuidad a la enseñanza superior, entre tantas otras cosas.

Recién en el 2015 la Argentina fue el primer país de América latina con una ley, no un decreto (que podía sustituirse por otro decreto), que garantiza la gratuidad de la formación de grado, el ingreso irrestricto y refuerza la autonomía universitaria y en el caso de la UNLP, deroga el espíritu la esencia de ese fatídico art. 50 que llevaba a que las facultades pudieran definir su propio sistema de ingreso por encima del cogobierno de la universidad, el consejo superior y la asamblea universitaria, lo que nos llevó a un conflicto de casi dos décadas con la facultad de medicina en dos conceptos de universidad : uno inclusivo y masivo y otro selectivo y privatista. 
Redea: En ese marco la UNLAM (Universidad Nacional de La Matanza) y la UNSAM (Universidad Nacional de San Martín) iniciaron dos acciones judiciales para declarar la inconstitucionalidad del art. 2 de la nueva Ley y el art. 4 en referencia al ingreso irrestricto y la imposibilidad que tienen las facultades de poder decidir algún sistema de selección. Esto tuvo, tanto en primera instancia como en la alzada, una declaración de inconstitucionalidad. Con el cambio de gobierno a partir del 2015, el Estado Nacional dejó firmes esas decisiones por no apelarlas, con lo cual, quedó firme la declaración de inconstitucionalidad por la omisión en la continuidad del proceso por parte del estado y del actor, que eran las universidades. Esto llegó a la Corte Suprema de Justicia por una insistencia de la Procuradora General de la Nación, que tiene la representación del interés general de la sociedad en juicio, quien insistió en la constitucionalidad de la ley. Esto está en estado de decidirse en la Corte. ¿Cuál es la opinión en particular de la UNLP y el resto del CIM (Consejo Interuniversitario Nacional).

No solo dos universidades, también fue la universidad de Río Negro... yo creo que es toda una paradoja. Tanto desde el CIM como de cada una de las universidades, el concepto de universidad pública en función y al servicio de la sociedad, siempre interpretó que el contrato reformista era que el ascenso social se produjera a partir de la masividad en el acceso al conocimiento. Con lo cual resulta incómodo, sospechoso, que universidades públicas, que además representan intereses populares, puedan judicializar una decisión de gratuidad generalizada o de igualdad de oportunidades para acceder a la educación superior. Estas son conquistas de las cuales no deberíamos retroceder, conquistas de una sociedad que busca ser igual, que busca en las definiciones de justicia social hechos concretos como este, que es la oportunidad de acceder. La inclusión no significa que uno resigne la contraparte del contrato: uno siempre exige que estos beneficios sean para aquellos que tienen la voluntad 
de hacer el esfuerzo de estudiar y recibir una educación de calidad. Con lo cual el sistema es reciproco. Nosotros damos las facilidades y oportunidades y pretendemos recibir el esfuerzo de cada uno de los individuos que se lleva, como resultado final un título universitario que le permite tener más oportunidades e inserción social con la expectativa de progreso. Así de simple fue el pensamiento del 18 , de la reacción ante la universidad clerical de Córdoba. Esa mezcla de criollos y de inmigrantes que conformaban la sociedad de la época, distinguió en la educación la posibilidad de a partir del esfuerzo individual tener más posibilidades de construir una sociedad más igual, con más parecidos entre los extremos. La universidad en eso es una herramienta fundamental de oportunidades de progreso y desarrollo generalizado y de construcción de soberanía, es difícil de ver a una universidad que esté encontrándole la vuelta para vulnerar esos principios, esos postulados fundamentales.

\section{Redea: Pensando en nuestra universidad... en los últimos días se realizaron elecciones en las distintas unidades académicas. ¿Que opinión le ha merecido este proceso elec- cionario?}

Nosotros estamos muy satisfechos con este proceso. Creo que la universidad, como todas las instituciones en democracia, va dando sus pasos hacia una mayor madurez. Tuvimos una etapa adolescente, que todos transitamos con encendidas disputas aun sin distinguir las diferencias de modelo. Nos fuimos enfocando en una condición que dejara más claro cuáles eran las diferencias en las que no podíamos claudicar, el modelo de universidad que pretendíamos construir, y me parece que eso le fue sacando dramatismo. La disputa enriquece, las mayorías y las minorías no significan enemigos irreconciliables, el pensamiento diferente es una condición que la universidad defiende encendidamente. Nosotros creemos que en la universidad, desde los distintos matices y miradas, se puede encontrar un lugar de convergencia en cuanto a para que estamos y que 
queremos ser como institución publica y al servicio de nuestro pueblo y en eso pueden convivir distintas ideas. Creo que si hay una universidad que es reactiva al pensamiento único es la universidad pública, eso del avasallamiento, eso de hegemonizar en un único discurso ha sido un tema que ha encontrado una coraza en nuestra universidad y hemos defendido esa condición. Por eso las elecciones son un hecho democrático casi apológico, el sistema de cogobierno es el espejo de lo que más puede conseguir un sistema democrático: todos los claustros, todos los niveles, todos representan, todos producen la síntesis de una elección, sea en su unidad académica o en los propios colegios de la universidad, sea en la Asamblea Universitaria.

Esta es una universidad que a partir de la reforma del estatuto del año 2008 tiene participación de los cuatro claustros, más los colegios universitarios de pregrado y eso significa un infinito nivel de representación. Haber incorporado al sector no docente al cogobierno fue todo un avance en la concepción política de la universidad. Poder realizar ese ejercicio cada cuatro años es un hecho celebrable y la verdad que no ha tenido grandes estridencias. Se ven en algunas unidades académicas procesos que se agotan por el propio sistema democrático y que le da oportunidad a una renovación, es el caso de medicina por ejemplo.

Redea: Haciendo un balance de los últimos años, ¿que logros podría destacar de nuestra Universidad y cuáles serían los desafíos que aún quedan pendientes de cara al futuro?

La universidad funciona con un plan estratégico desde el año 2004, plan que dirijo desde ese momento. Esa universidad transitó durante esos 14 años un proceso de satisfacción de demandas insatisfechas de grandes dimensiones. La UNLP había crecido tremendamente, tenía 40 mil integrantes cuando empezó la democracia y hoy tenemos 160 mil, con lo cual todo le quedaba chico. Necesitábamos metros cuadrados, presupuesto, equipamientos, cargos docentes, no docentes, todo esto fue tomando otra dimensión a medida que fuimos dando pasos 
que consensuamos permanentemente en el debate político. Es innegable reconocerle el crecimiento edilicio, el desarrollo que ha tenido. Hemos duplicado la cantidad de docentes, se han consolidado en un estándar superior los salarios tanto docentes como no docentes, eran irrisorios en el principio de la década pasada, la gente venía a dar clases por el IOMA [obra social], hoy existe la carrera universitarias, hoy, aun desde facultades profesionalistas como la nuestra, hay intenciones de quedarse en la universidad, de investigar, de hacer una carrera universitaria, de hacer extensión, de hacer transferencia.

La UNLP es la universidad más compleja que tiene la Argentina, la UBA es la más grande, nosotros somos la más compleja. El balance es altamente positivo en cuanto a las metas que nos propusimos y el estado en el que está hoy, lo cual nos genera el desafío en pensar menos en las demandas insatisfechas y pensar más en las responsabilidades que tenemos que asumir a futuro, porque antes no teníamos aulas, ahora las tenemos, no teníamos laboratorios, ahora los tenemos, no teníamos cargos, ahora los tenemos. Debemos diseñar políticas académicas que efectivamente verifiquen que somos una herramienta de transformación y de desarrollo: el rendimiento académico, el crecimiento en el egreso, el sostenimiento de este proceso, que pasa a ser un desafío fundamental. Nosotros hoy tenemos todavía una gran cantidad de estudiantes que no llegan a probar dos materias al año, que no serían alumnos regulares, a pesar de ser una de las universidades públicas que tiene un estándar mayor. Aún tenemos relaciones que nos dejan muy lejos de la satisfacción, debemos explicarle a nuestra sociedad que de cada diez pibes que ingresan se reciben tres... y en el país el promedio es que se reciben dos: nuestro objetivo es que se reciban cinco, que por lo menos se reciban la mitad, no queremos parangonarnos ni con Finlandia ni con Canadá, porque nosotros además venimos de una educación pública gratuita, son otras condiciones, pero si queremos estar al nivel de otros países centrales como el propio Estados Unidos, 50\% en la relación de ingreso/egreso. 
Creemos que tenemos que asumir una responsabilidad particular en retener nuestros recursos humanos formados. El año pasado tuvimos situaciones incomodas en el rechazo de aspirantes de ingreso a carrera en el Conicet que ya eran doctores, que ya habían sido admitidos, que habían pasado por los requerimientos y que sin embargo se quedaron afuera. El capital humano formado es el capital más importante que puede producir la universidad como una institución que produce conocimiento, porque producir conocimiento es producir soberanía, es no tener que comprar afuera lo que podemos producir nosotros, con lo cual el tema tiene su cadena. No hay que analizarlo en una linealidad corta, sino en una proyección integral de cuál es el proyecto de desarrollo de este país. Para nosotros es central la retención de esos recursos humanos formados, de poder ofrecerles una mayor dedicación exclusiva, al menos por un tiempo, después que termina su doctorado. El que estudia el doctorado es porque quiere tener una carrera de investigación, y si los organismos de investigación no lo dejan entrar, no tiene retorno, se queda en un limbo o se tiene que ir al exterior. Esas son las cosas que hemos reclamado sin distinción de alineamiento político. Recuperemos los recursos que perdimos y sobre todo no dejemos ir los que tenemos formados y estamos formando, que son una inversión de toda la gente durante tantos años. La universidad tiene que tener su propia política de colaborar, no podemos suplantar a sectores supremos del Estado, pero si darle una señal a nuestros propios graduados de que nosotros pensamos en ellos y los pensamos en una dimensión estratégica, no solidaria. La solidaridad no es misericordia, nosotros pensamos en una sociedad de iguales, por eso pensamos en una sociedad solidaria, no misericordiosa de los que más tienen con los que menos tienen, que es naturalizar la diferencia. Por lo tanto nuestras políticas de extensión tenemos que impulsarlas en procesos formativos más que en la circunstancia de estar presente para resolver un hecho de necesidad, no porque no sea valioso, pero nuestra centralidad esta en darle elementos perdurables a esa sociedad, a esa comunidad 
que no está buscando en ser ingeniero ni filosofo, sino que lo que está buscando es tener herramientas para poder tener más oportunidades de progresar, de insertarse en el mundo del trabajo. Para pasar de una universidad pública a popular, que es lo que buscamos, necesitamos que este concepto esté presente, necesitamos construir redes, no en el estándar "marquetinero" del fenómeno global, sino en la condición de identificar a aquellos que tienen nuestros mismos objetivos, nuestros mismos problemas y nuestras mismas necesidades de fortalecer a nuestra sociedad. Desde ya que tenemos que integrarnos el mundo, aprovechar todas las oportunidades de vínculos, fortalecer procesos sustentables, alianzas estratégicas, porque estamos construyendo los mismo, con otras universidades, con otras instituciones, con los graduados... tenemos que consolidar la figura emprendedora de nuestros graduados creando logísticas que ninguno individualmente puede construir ni acercarse, $y$ ponerla a disposición de todos:... estamos poniendo en marcha un "centro de la madera" que tiene que estar al servicio de los ingenieros forestales, estamos poniendo en marcha un "centro informático", que tiene que juntar las microempresas que hoy conviven en La Plata en locales, casas de familia, y que puedan usar todo el potencial de la universidad...terminamos un "set de cine" que es el más moderno de América Latina para el desarrollo de industrias culturales, fijamos convenios en ese sentido para que esta ciudad, que tiene ese perfil universitario, de conocimiento y que tiene más de cien mil graduados activos viviendo en la región, pueda tener un efecto sinérgico que conlleve al desarrollo colectivo, esos son procesos de arrastre. 